\title{
AVALIAÇÃO DA USABILIDADE DE INTERFACES DE SISTEMAS VGI NA TAREFA DE INSERÇÃO DE FEIÇÕES.
}

\author{
Usability Assessment of VGI Systems Interfaces in Feature Insert Task.
}

\author{
Péricles Luiz Picanço Jr. ${ }^{1}$ \\ Luciene Stamato Delazari ${ }^{1}$ \\ ${ }^{1}$ Programa de Pós-Graduação em Ciências Geodésicas - Universidade Federal do Paraná \\ Curitiba, Paraná, Brasil. Email:pericles.picanco@outlook.com, luciene@ufpr.br
}

\section{Resumo:}

Um sistema de mapeamento voluntário (VGI - Volunteer Geographic Information), é uma aplicação computacional na qual um indivíduo voluntariamente visualiza, organiza, e dissemina livremente dados e informações geográficas que podem ser usadas gratuitamente por qualquer usuário (Tulloch, 2008). O objetivo principal de um sistema VGI é permitir que um voluntário colete dados geográficos; para isso é essencial considerar, durante o processo de desenvolvimento da aplicação, a interação do usuário com os elementos da interface. Sistemas VGI como o OpenStreetMap têm diferentes processos de interação com o usuário, os quais implicam em diferentes tipos de interfaces, funcionalidades e formulários. A execução de uma tarefa, assim como o seu resultado, pode diferir dependendo do sistema utilizado. Diferentes processos de interação acarretam em diferentes experiências do usuário, e consequentemente afetam a qualidade e a confiabilidade da informação espacial coletada. $O$ processo de interação pode também influenciar a retenção de usuários no sistema, bem como a adesão de novos voluntários. O objetivo desta pesquisa é obter um panorama de aspectos da usabilidade de sistemas VGI, especificamente na tarefa de inserção de feições geográficas, desde o acesso ao sistema até o sucesso ou falha na execução da tarefa. Para execução desta pesquisa foram realizados testes com usuários com diferentes tipos de familiaridade com os sistemas Wikimapia, OpenStreetMap, e Wikiloc, com o objetivo final de inserir uma ou mais feições geográficas nesses aplicativos.

Palavras-chave: VGI. Usabilidade. Experiência do usuário.

\section{Abstract:}

A volunteered geographic information system (VGI) is a computer application which allows an individual voluntarily visualizes, organizes, and disseminates geographical data and information for free use by any user (Tulloch, 2008). The main goal of a VGI is to obtain volunteered geographical data, so it is essential to consider the user's interaction with all the interface elements in its development. VGI systems such as OpenStreetMap have different interaction processes with the user, which entail different types of interfaces, functions, and forms. The 
execution of the same task can then be processed and performed in different ways, depending on the system used. Different interaction processes can lead to several user experiences, and affect the quality of the geographical information collected. The interaction process can also influence maintenance of active users and the addition of new volunteers. The aim of this research is therefore to obtain an overview of the usability of VGI systems, specifically in terms of geographical features insertion, from system access until the success or failure of the task execution. To achieve this, we asked users with different levels of familiarity with Wikimapia, OpenStreetMap, and Wikiloc to execute a set of tasks with the objective of inserting one or more features in these systems.

Keywords: VGI. Usability. User's experience

\section{Introdução}

Em um país de tamanho continental como o Brasil, o crescimento de sistemas de mapeamento voluntário traz possibilidades concretas de uso de bases de dados espaciais colaborativas pela população e de associar a Informação Geográfica Voluntária às informações de órgãos oficiais. Informação Geográfica Voluntária vem do termo em inglês Volunteered Geographic Information (VGI), e refere-se a toda a informação espacial que foi disponibilizada colaborativamente, voluntariamente e gratuitamente, através de em um sistema computacional desenvolvido especificamente para a livre inserção e uso dessa informação espacial.

Sistemas de mapeamento voluntário já foram adotados pela administração pública no Brasil como fonte de feedback do cidadão sobre a dinâmica urbana, para o mapeamento de áreas de risco social e de ocupação irregular. Exemplo disso é a plataforma 1746 da Prefeitura da Cidade do Rio de Janeiro, na qual o cidadão pode marcar no mapa, através de um aplicativo móvel, incidentes na cidade. Outro exemplo é o projeto "Na área" (http://www.techtudo.com.br/noticias/noticia/2014/05/microsoft-lanca-o-bing-na-area-projeto-da-primeiropasso-no-morro-do-vidigal.html) parceria da Microsoft e a Prefeitura do Rio de Janeiro, que através de um aplicativo móvel, desenvolvido via plataforma BingMaps, os moradores das favelas cariocas podem mapear as ruas e locais em suas comunidades, armazenando a informação em seu serviço de mapas.

O crescimento do uso dessas ferramentas, combinado a falta de pesquisas sobre a usabilidade especificamente de sistemas VGI (Parker, 2014), traz a necessidade do desenvolvimento de pesquisas que abordem o desenvolvimento de interfaces e sistemas de mapeamento colaborativo, com foco nos usuários que diferem do usuário tradicional de Internet. Faz-se necessário avaliar alternativas inclusivas, adequadas aos processos cognitivos destes usuários, permitindo o desenvolvimento de interfaces de usabilidade específicas a um grupo de usuários.

A partir disso, nesta pesquisa tem-se o objetivo de realizar a análise da usabilidade de sistemas VGI, através do uso das Heurísticas de Usabilidade (Nielsen, 1993), durante as tarefas de inserção de feições geográficas. Objetiva-se também a identificar os elementos de interface dos sistemas VGI testados que influenciam a forma como os usuários interagem com as interfaces e consequentemente influenciam diretamente no seu uso e adoção.

Este trabalho foi desenvolvido a partir da avaliação de três sistemas de mapeamento voluntário, atualmente em uso na Internet, o OpenStreetMap (OSM), o Wikimapia e o Wikiloc. Os sites foram escolhidos pela abrangência de operação, número de usuários e a existência de 
funcionalidades semelhantes. Par a o teste e a análise de usabilidade foram elaboradas tarefas, focadas na inserção de feições, para cada um dos sites.

Assim, foi solicitado a um grupo de 30 pessoas que inserissem feições geográficas através de um dos três sistemas escolhidos e respondessem a questionários relacionados às tarefas executadas. A partir da análise dos testes, questionários e gravações, foi possível avaliar e classificar cada sistema em relação as Heurísticas de Usabilidades e constatar os elementos de interface que podem ser melhorados nos sistemas e de uma maneira geral poderiam ser considerados na construção de novas aplicações.

Parte desta pesquisa foi apresentada no International Cartographic Conference 2015, realizado em agosto de 2015 na cidade do Rio de Janeiro, Brasil.

\section{Sistemas de Mapeamento Voluntário}

O termo Volunteered Geographic Information (VGI) refere-se a um sistema de mapeamento colaborativo no qual a informação geográfica é criada e atualizada através adesão e do trabalho voluntário de usuários, não necessariamente especialistas. É derivado do conceito chamado crowdsourcing, introduzido com a WEB2.0 (O'reilly, 2005), no qual o usuário consome e cria conteúdo em um ambiente colaborativo (Goodchild, 2007).

Tulloch (2008) definiu os Sistemas de Informação Geográfica Voluntária como uma aplicação computacional, na qual um indivíduo voluntariamente visualiza, organiza e dissemina dados espaciais e informações geográficas para o uso livre de qualquer indivíduo. O uso dessas aplicações varia entre os usuários e um sistema projetado para um usuário acostumado com a linguagem de grandes portais de informação ou softwares comerciais pode não atender as necessidades de um usuário de Internet menos experiente (Nielsen \& Loranger, 2007).

\subsection{Sistemas VGI Testados}

Na execução desta pesquisa foram escolhidos três sistemas VGI independentes e em operação na Internet. O OpenStreetMap (https://www.openstreetmap.org/, Wikimapia (http://wikimapia.org/) e o Wikiloc (http://www.wikiloc.com/), cujas interfaces encontram-se na Figura 1. Os sistemas são semelhantes em número de usuários, abrangência geográfica e tarefas, sendo o site Wikiloc o mais destoante entre os três, pois tem um público mais específico (em objetivo e não em experiência), mas a sua comparação é válida no contexto funcional e de uso.

O sistema OpenStreetMap (OSM) consiste em um esforço internacional para criar um mapa com dados para uso livre, desenvolvido através do trabalho voluntário e gratuito. $\mathrm{O}$ usuário pode baixar livremente as bases de dados espaciais e através de login e senha, criada gratuitamente, pode inserir ou atualizar qualquer dado geográfico no sistema. A principal vantagem no dado gerado no OSM é a velocidade de atualização da informação e a possibilidade de encontrar 
informações diferentes das disponibilizadas ou vendidas nos países de origens dos usuários (Haklay, 2010).

OPENSTREETMAP

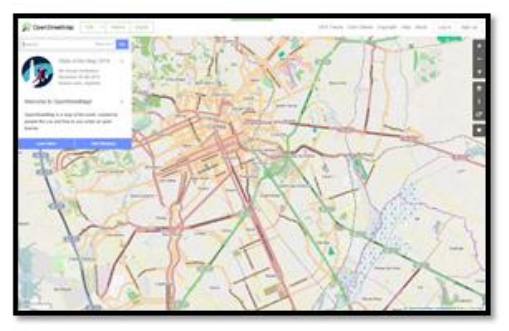

http://www.openstreetmap.org/
WIKIMAPIA

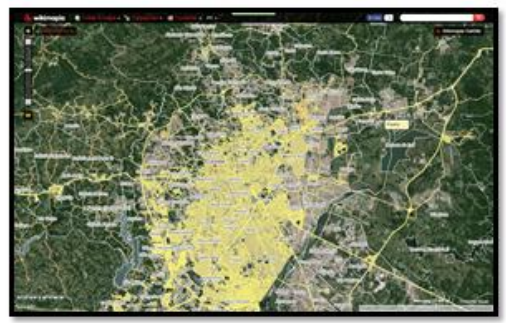

http://wikimapia.org/
WIKILOC

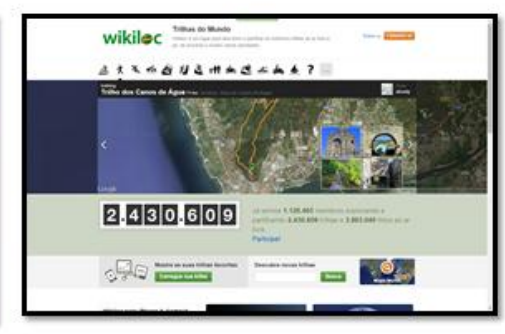

http://www.wikiloc.com/

Figura 1: Interfaces dos sistemas OpenStreetMap, Wikimapia e o Wikiloc.

O Wikimapia surgiu em 2006 com a proposta de criar um mapa colaborativo de conteúdo aberto, no qual qualquer pessoa pudesse inserir feições geográficas e atributos, partilhando seus conhecimentos do território. É atualizado em todo o mundo através da inserção de objetos geográficos e seus atributos, proporcionando o livre acesso aos dados de domínio público. O objetivo do site é ser claro, em constante atualização e simples a qualquer pessoa, mesmo sem experiência prévia (Wikimapia, 2012).

O Wikiloc foi criado em 2006, na Espanha, com o objetivo de compartilhar facilmente trilhas de GPS e pontos de interesse de todo o mundo. A sua evolução e popularidade foi crescente desde então e hoje o sistema web Wikiloc está traduzido para 21 línguas e disponibiliza além das feições geográficas, correspondentes às trilhas com múltiplas finalidades, fotos e vídeos com marcações geográficas.

Os números de usuários nos três sistemas, em outubro de 2014, eram: o OSM possuía cerca de 1,8 milhões de usuários, enquanto o Wikimapia tem cerca de 2 milhões de usuários e o Wikiloc, o menor em estrutura e o mais específico em objetivos, tem 1,6 milhões de usuários.

\section{Usabilidade e Heurísticas de Avaliação}

A usabilidade mede a facilidade do usuário em completar objetivos específicos com eficácia, utilizando um produto projetado para proporcionar eficiência e satisfação ao usuário, em um contexto específico (ISO 9241-11). Existem cinco componentes de qualidade da usabilidade: aprendibilidade, eficiência, memorabilidade, erros e satisfação (Nielsen, 1993, p.26).

A usabilidade é um aspecto importante na construção de sistemas de informação e websites, visto que a primeira experiência do usuário é determinante para o seu retorno. A dificuldade de um usuário encontrar o que deseja, de navegar nas páginas e menus ou perder tempo tentando entender uma interface, afugenta esse usuário que procurará opções mais intuitivas e agradáveis (Nielsen \& Loranger, 2007).

Em um workshop entre especialistas em usabilidade, Brown et al. (2013) elencaram os temas considerados desafios da usabilidade para a informação geográfica. Um dos temas foi chamada de "Novas direções para o uso da informação geográfica", do qual se destaca a informação 
voluntária geográfica. Nesse tema os especialistas pontuaram que o encorajamento dos usuários a colaborar na coleta de dados geográficos traz uma série de desafios para o design da informação geográfica.

A partir da afirmação de Goodchild (2008), que talvez um determinante para a seleção dos dados é a acessibilidade à eles, mais que a sua qualidade geométrica, os mesmos especialistas consideraram que as ferramentas disponíveis para a manipulação dos dados, a sua compatibilidade e consistência com outras ferramentas de desenvolvimento podem ser fatores importantes que impactam o uso da informação geográfica. Segundo Haklay et al. (2008), apesar de todos os avanços nos Sistemas de Informações Geográficas, não há nada realmente novo, apenas a interatividade online.

Para Parker (2014), apesar de alguns autores terem categorizados os usuários associados aos sistemas VGI, a relação entre o usuário e as suas percepções sobre esses sistemas, útil no contexto da construção de uma interface centrada no usuário, não é discutida na literatura atual. Ainda segundo o autor, é necessário um aprofundamento nas pesquisas sobre como os aspectos específicos de usabilidade afetam o projeto de sistemas VGI.

A partir do exposto, fica evidente que a usabilidade da informação geográfica decorrente de seus novos usos se baseia nas formas de disponibilização e acesso, assim como nos aspectos de usabilidade das funções e interfaces com o usuário.

No estudo da usabilidade os testes com usuários são fundamentais para obter dados do uso de interfaces de aplicações específicas (Nielsen, 1993), pois permitem identificar problemas concretos na interação do usuário com o sistema. O autor ainda relaciona quatro etapas de um teste: preparação, introdução, o teste propriamente dito e o debriefing.

O debriefing é um questionário aplicado pelo moderador do teste após o seu término e sempre antes de qualquer tipo de discussão a respeito das atividades ou do sistema testado. Através dele é possível entender as impressões dos usuários sobre o sistema, assim como o nível de satisfação com a experiência de uso (Nielsen, 1993).

Nielsen (1993) relaciona os métodos de testes de usabilidade de uso comum, como a mensuração de desempenho, o Think Aloud, a observação direta, a avaliação de heurísticas, a avaliação feita por especialistas, os questionários e a inspeção formal. Neste trabalho optou-se pela combinação dos métodos dos testes mensuração de desempenho e questionários, aplicados diretamente aos usuários, com foco na completude das tarefas pedidas e na experiência dos usuários.

A mensuração de desempenho é um dos métodos base para a pesquisa em fatores humanos e igualmente importante na pesquisa e avaliação de usabilidade de sistemas. Nesse método é medido o desempenho dos usuários através da execução de um grupo de tarefas pré-definidas e simultaneamente são coletadas informações de tempo e erros (Nielsen, 1993). Também são avaliados nesse método o uso de manuais e funções de ajuda, bem como considerações subjetivas sobre o usuário, como frustrações e experiências bem-sucedidas (Mendonça, 2009).

$\mathrm{Na}$ tarefa de explicar o grande número de problemas observados no desenho das interfaces de usuários, Nielsen (1993) elaborou as Heurísticas de Usabilidade, que são princípios básicos de usabilidade e podem ser utilizados para o projeto ou avaliação de interfaces. Neste trabalho, as Heurísticas de Usabilidade foram utilizadas como guia para a avaliação final dos sistemas VGI testados. Na Tabela 1estão relacionadas se as Heurísticas de Usabilidade e suas descrições de acordo com Nielsen (1995) e Cybis (2010). 
Tabela 1 : Heurísticas de Usabilidade. Fonte: (Nielsen, 1993) \& (Cybis, 2010)

\begin{tabular}{|c|}
\hline $\begin{array}{l}\text { Visibilidade do estado do sistema } \\
\text { O sistema deve sempre manter os usuários informados sobre o que acontece no sistema, através de } \\
\text { avisos apropriados e em tempo hábil. }\end{array}$ \\
\hline Mapeamento entre o sistema e o mundo real \\
\hline $\begin{array}{l}\text { O sistema deve usar a linguagem dos usuários, com palavras, frases e conceitos familiares, em vez } \\
\text { de termos técnicos. Seguindo convenções do mundo real, fazendo as informações aparecerem em } \\
\text { uma ordem natural e lógica. }\end{array}$ \\
\hline Liberdade e controle do usuário \\
\hline $\begin{array}{l}\text { Os usuários frequentemente escolhem as funções do sistema acidentalmente e deve haver uma } \\
\text { indicação clara de saída da função indesejada sem ter que migrar de tela. Suporte com as funções de } \\
\text { undo e redo. }\end{array}$ \\
\hline Consistência e Padrões \\
\hline $\begin{array}{l}\text { Os usuários não devem ter que supor se palavras diferentes, situações ou ações significam a mesma } \\
\text { coisa. Seguir convenções da plataforma. }\end{array}$ \\
\hline Prevenção de erros \\
\hline $\begin{array}{l}\text { Melhor do que boas mensagens de erro é um projeto cuidadoso que impeça um problema ocorrer } \\
\text { em primeiro lugar. Eliminar as condições passiveis de erros ou verificá-los e apresentar aos usuários } \\
\text { uma opção de confirmação antes de se comprometer com a ação. }\end{array}$ \\
\hline Reconhecer em vez de relembrar \\
\hline $\begin{array}{l}\text { Minimizar a carga de memória do usuário, fazendo objetos, ações e opções visíveis. O usuário não } \\
\text { deve ter que lembrar informações de uma parte do diálogo para outra. Instruções de utilização do } \\
\text { sistema devem estar visíveis ou facilmente recuperáveis sempre que necessário. }\end{array}$ \\
\hline Flexibilidade e eficiência de uso \\
\hline $\begin{array}{l}\text { Aceleradores podem frequentemente melhorar a interação para todos os usuários do sistema, mesmo } \\
\text { que sejam invisiveis aos usuários iniciantes. }\end{array}$ \\
\hline Design estético e minimalista \\
\hline $\begin{array}{l}\text { Os diálogos não devem conter informações irrelevantes ou raramente necessárias. Diminui a } \\
\text { visibilidade relativa dos elementos da interface. }\end{array}$ \\
\hline Suporte para o usuário reconhecer, diagnosticar e recuperar erros \\
\hline $\begin{array}{l}\text { Mensagens de erro devem ser expressas em linguagem simples (sem códigos), indicar com precisão } \\
\text { o problema e construtivamente sugerir uma solução. }\end{array}$ \\
\hline Ajuda e documentação \\
\hline $\begin{array}{l}\text { Qualquer informação deve ser fácil de pesquisar, focada na tarefa do usuário, listar passos concretos } \\
\text { a serem realizados, e não ser muito grande. }\end{array}$ \\
\hline
\end{tabular}

\section{Metodologia}

Para o desenvolvimento desta pesquisa, a metodologia deste trabalho foi executada conforme as etapas dispostas na Figura 2: 


\begin{tabular}{|c|l|}
\hline Etapa 1 & • Análise baseada em Heurística \\
\hline Etapa 2 & • Definição de Tarefas, Questionários e Métricas \\
\hline Etapa 3 & • Execução dos Testes \\
\hline Etapálise dos Testes 4 & - Avaliação da Interface por meio de Heurísticas \\
\hline Etapa 5 &
\end{tabular}

Figura 2: Etapas da Metodologia

\subsection{Etapa 1 - Pré-teste: Análise baseada em heurística}

Nessa etapa da metodologia, os sites escolhidos para os testes foram analisados com base nos seus fluxos de interação para a identificação de possíveis pontos de atenção, nos quais o usuário pudesse ter algum problema de comunicação e/ou usabilidade. Para isto, os sites passaram por testes que simularam o uso real das suas funcionalidades, resultando em três fluxogramas que retratam o processo ideal que um usuário deve executar para inserir uma feição geográfica.

A partir desses fluxogramas, foram utilizadas as Heurísticas de Usabilidade para a identificação das possíveis falhas no processo de iteração com o usuário, assim podendo considera-las durante a elaboração das tarefas e identificar suas consequências nos resultados dos testes. As heurísticas ainda serviram para a avaliação final dos sites, numa tentativa de identificar qual deles utilizou com melhor propriedade as diretrizes de usabilidade.

\subsection{Etapa 2: Definição de Tarefas, Questionários e Métricas}

A etapa 2 da metodologia corresponde a definição das tarefas, questionários e métricas utilizadas pelos usuários para graduar a dificuldade e satisfação no uso dos três sistemas. A tarefa principal do teste de usabilidade foi a de inserção de feição geográfica, por isso foram definidas para cada site uma tarefa correspondente, se baseando nos fluxos de processo desenvolvidos na etapa anterior.

Essas tarefas foram definidas baseadas nos fluxogramas elaborados, assim foi possível enunciar as tarefas do teste de forma objetiva, suprindo os usuários testados de todas as informações necessárias para a execução das tarefas, tais como: usuários, senhas e nomenclaturas adequadas.

Os itens desenvolvidos nesta etapa foram:

1. Questionário A - Informações sobre o usuário e comportamento de uso de Internet e mapas.

2. Tarefa 1 - Conhecer e explorar o site de teste.

3. Questionário B - Impressões iniciais sobre o site.

4. Tarefa 2 - Inserir uma feição geográfica pré-definida e local informado.

5. Tarefa 3 - Editar a feição geográfica inserida na Tarefa 2. 
6. Tarefa 4 - Apagar a feição geográfica inserida e editada.

7. Questionário C - Impressões sobre as Tarefas 2, 3 e 4.

\subsubsection{Questionário $A$}

O Questionário A foi aplicado a todos os usuários antes de executarem quaisquer das atividades. Ele coleta informações sobre os perfis dos usuários, como: faixa etária, nível de escolaridade, uso de Internet, uso de dispositivos móveis e de mapas na Internet. Não foram coletadas informações de cunho pessoal que permitissem a associação dos questionários à identidade dos usuários.

As informações coletadas através deste questionário ajudaram a entender o conhecimento prévio que os indivíduos testados possuíam e como esse conhecimento pode influenciar a interação dele com o sistema. Todas as perguntas neste questionário eram objetivas, permitindo que os indivíduos testados escolhessem desde respostas binárias como "Sim" ou "Não", até expressões que retratassem uma frequência, como: nunca, raramente, às vezes, e sempre.

\subsubsection{Tarefa 1}

Segundo Nielsen (2007), somente os sites que conseguem reter os usuários nos primeiros 30 segundos, têm chance de fixá-los ao conteúdo por 2 minutos ou mais, o que, segundo o autor, é uma eternidade para a Internet. Isso evidencia o desafio e a importância da construção de interfaces que consigam comunicar seus objetivos e seus diferenciais aos usuários. No contexto de um sistema VGI, a comunicação de valores e objetivos em um curto prazo de tempo é ainda mais crítica, pois pode ser a porta de entrada para um novo voluntário e consequentemente a novos conteúdos geográficos.

$\mathrm{Na}$ tarefa 1, os usuários foram solicitados a acessar um dos sistemas VGI e explorá-lo por 5 minutos. Baseado no trabalho de Nilsen (2007), no qual o tempo mínimo de fixação com sucesso ao conteúdo foi de 2 minutos, foi estipulado o tempo máximo de 5 minutos para a primeira interação do usuário com os sistemas. Assim, os indivíduos testados tiveram tempo suficiente para explorar os sistemas com conforto, mas curto o suficiente para que os indivíduos testados não perdessem o foco.

Nessa tarefa os usuários foram orientados a agir livremente durante o tempo determinado, sem um roteiro ou tarefa específica, como fariam ao acessar a primeira vez um novo site. O tempo foi monitorado pelo facilitador do teste, que avisou aos usuários o seu término e solicitou que os usuários testados respondessem as questões apresentadas no Questionário B.

\subsubsection{Questionário B}

O objetivo do Questionário B foi obter as impressões iniciais dos usuários testados e quais informações eles tiveram acesso nesse período. Os usuários foram perguntados, entre outras coisas, sobre qual o objetivo dos sites, se as informações dispostas eram claras, qual o público alvo e se já o conheciam os sistemas. Dessa forma pudemos visualizar a primeira impressão dos usuários testados em relação aos sistemas e se a informação disposta era evidente o suficiente 
para que o usuário a utilizasse e possivelmente justificando alguns dos resultados das tarefas executadas.

\subsubsection{Tarefa 2}

A Atividade 2 é a tarefa principal e o objetivo do teste de usabilidade. Nela os usuários foram solicitados a inserir uma feição em um dos sites. Foram escolhidas três feições geográficas, no Campus do Centro Politécnico da UFPR: o prédio do grupo escoteiro universitário para o site OSM, o estacionamento em frente a piscina para o Wikimapia e um circuito nas vias do campus como uma trilha para o site Wikiloc. Junto ao enunciado da atividade apresentou-se uma figura de localização da região (Figura 3) e um mapa mostrando exatamente a feição geográfica a ser inserida (Figura 4), assim como se esperava o resultado final.

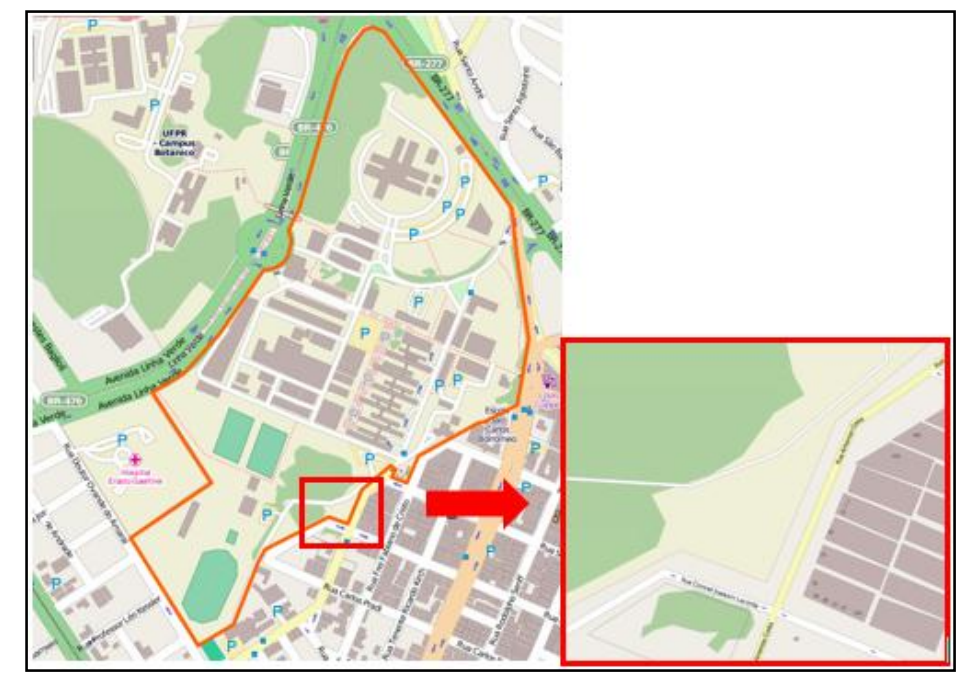

Figura 3 : Localização da região de teste
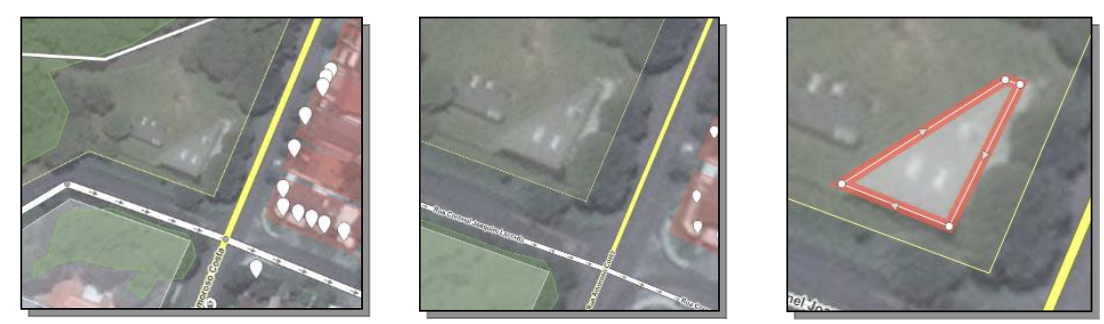

Figura 4 : Localização da feição e resultado esperado na interface do site testado.

Além das informações sobre a localização geográfica e atributos necessários para a inserção da feição teste, o enunciado da tarefa informou os dados de login e senha necessários para a autenticação em cada um dos sites. Não foi informado ao usuário quando e se os dados de login e senha seriam utilizados durante a execução das tarefas, o objetivo disso foi o de verificar se a autenticação de usuário seria uma barreira no processo de edição, como sugeria a análise do sistema.

A finalização da tarefa acontecia com o salvamento da feição, pois seria utilizada na continuação dos testes. Dessa forma, caso o usuário não completasse a execução da tarefa solicitada, ele não conseguiria prosseguir com o teste e seria necessário a repetição do processo ou a chamada do facilitador do teste. 


\subsubsection{Tarefa 3}

Na tarefa 3, os usuários foram solicitados a modificar as feições inseridas na tarefa 4.2.4. Foi disponibilizado ao usuário um croqui com a mudança pretendida, mas foi deixado claro que o formato da feição não era o objetivo principal da tarefa e diferenças poderiam acontecer e não influenciariam o resultado. Novamente não são informados ao usuário quais funcionalidades ou sequência exata de ações que deveriam ser tomadas.

O principal objetivo desta tarefa foi verificar o uso das diferentes funcionalidades do site, complementares as funções de desenho e inserção de feições. Em um processo real de colaboração de informação geográfica em sistemas VGI, é comum a edição de aspectos da feição, assim como a edição das informações atribuídas, bem como a exclusão esporádica de feições erroneamente inseridas ou deslocadas.

\subsubsection{Tarefa 4}

A tarefa 4 foi a última tarefa solicitada aos usuários. Nela os usuários deveriam apagar as feições inseridas e posteriormente modificadas, finalizando a sessão de edição nos sistemas testados. Em seguida, o usuário é convidado a finalizar o teste, respondendo ao Questionário C. O objetivo principal desta tarefa foi o de perceber se os usuários testados entenderiam o processo de edição e exclusão, complementares as funções de edição e necessários em um contexto real de uso dos sistemas.

\subsubsection{Questionário C}

O Questionário C traz questões sobre a experiência do usuário durante a execução das tarefas 2, 3 e 4 . O usuário tem à sua disposição uma escala numérica para que possa expressar um grau de dificuldade durante as atividades, como localizar o local de teste, nas solicitações feitas e no uso das funções de inserção. Nessa escala, que vai de 0 (zero) à 10 (dez), o número zero corresponde à Extremamente Fácil e dez corresponde à Extremamente Difícil. A compilação das respostas do universo de usuários testados, assim como o resultado de suas análises e conclusões estão dispostas na sessão de resultados.

\subsection{Etapa 3: Execução dos testes}

Após a elaboração das tarefas, questionários e métricas, os testes foram aplicados a um grupo de 30 usuários, sendo 10 usuários por site testado, com diferentes graus de conhecimento sobre os sites escolhidos, mas com um conhecimento básico e uniforme sobre Cartografia e informações geográficas. O teste foi elaborado de forma a se obter dados derivados da execução das tarefas solicitadas, as respostas dos questionários e as evidências das interações dos usuários com a interface via a captura da tela durante a execução do teste.

Foi utilizada a infraestrutura física do Laboratório de Cartografia, do Departamento de Geomática, da Universidade Federal do Paraná, composto por 11 computadores desktop DELL OPTIPLEX 790, com o sistema operacional Windows 7 - Ultimate 64bits, e acesso à Internet 
com uma velocidade de 10Mbits/s. Aos computadores foram acopladas câmeras digitais para a gravação da voz e imagem dos participantes através de um software de captura de tela.

A gravação das ações em tela e das reações dos indivíduos testados foram importantes para a verificação de possíveis dissonâncias entre as respostas dos questionários e os resultados efetivos das tarefas. Além disso, pode-se também entender a dinâmica no uso das ferramentas e elementos da interface que puderam ser utilizadas na análise dos testes.

Os testes foram aplicados em três ocasiões diferentes, decorrentes da disponibilidade dos participantes, com a duração aproximada de 40 minutos.

\subsection{Etapa 4: Análise dos testes}

O processo de análise dos resultados dos testes foi dividido em (i) análise e compilação dos resultados dos questionários, (ii) análise e sistematização das evidencias dos vídeos e (iii) cruzamento dos resultados das análises i e ii.

Para todas as tarefas foram desenvolvidas planilhas nas quais as informações foram compiladas e totalizadas, utilizando uma abordagem quantitativa para a geração de parâmetro e proporcionar a comparação entre os testes dos sites. Apesar disso, a avaliação da interface e as conclusões obtidas são de cunho qualitativo e evidenciam tendências e em especial evidencias de um possível comportamento de usuários em relação aos elementos de uma interface e de uma estratégia de usabilidade.

\subsection{Etapa 5: Avaliação da interface por meio de heurísticas}

As Heurísticas de Usabilidade, com mencionado anteriormente, podem ser utilizadas no projeto e avaliação de interfaces. Neste trabalho, foram utilizadas como guia para a avaliação final dos sistemas VGI testados. Essa avaliação permitiu que fossem elencados os pontos fortes e os possivelmente fracos dos sites testados, assim como pontuar possíveis mudanças de estratégia de interação nos sites citados e em novos projetos.

Baseando-se nas heurísticas e nos resultados dos testes com os usuários, definiu-se na Tabela 2 uma escala conceitual para uma avaliação holística dos sites. Essa escala parte da combinação dos sinais de positivo (+) e negativo (-), relacionando o símbolo matemático a mais ou menos características das heurísticas de usabilidade atendidas e constatadas nos três sistemas. Assim, a combinação dos símbolos caracteriza o atendimento às heurísticas como:

O resultado dessa avaliação foi disposto na sessão de resultados deste trabalho.

Tabela 2 : Classificação dos sistemas de acordo com as heurísticas de usabilidade.

\begin{tabular}{|c|c|}
\hline Insuficiente & -- \\
\hline Parcialmente & +- \\
\hline Totalmente & ++ \\
\hline
\end{tabular}




\section{Resultados}

O primeiro grupo de resultados obtidos durante o desenvolvimento desta pesquisa foram os fluxogramas de funcionalidades dos sistemas VGI escolhidos para o teste, esses fluxogramas evidenciam o fluxo de interação dos usuários com o site. Os sistemas OpenStreetMap, Wilkimapia e Wikiloc passaram por simulações de execução da tarefa de inserção de feições, afim de se obter o fluxo real de ações e decisões de um usuário ao utilizar suas funcionalidades.

Como exemplo, na Figura 5 pode-se ver o fluxograma desenvolvido para o site Wikimapia, nele existem dois fluxos de atividades separados (i) a inserção de feições sem a utilização de usuário e senha e (ii) o fluxo de desenho e edição para o qual é necessário a autenticação do usuário no sistema. Este fluxo demonstra a possibilidade de uma dissonância cognitiva associada a autenticação do usuário no sistema, a qual foi testada posteriormente. Os fluxos para os demais sites estão em anexo a este artigo.

O OSM conjuga as funcionalidades de inserção e edição de feições em um único fluxo de ações, favorecendo a aprendizado e a memorização do processo. O sistema exige a autenticação do usuário para que este possa executar qualquer tarefa no sistema, mas não limita tipos de feições ou atributos a partir do tempo de cadastro. O OSM ainda permite ao usuário criar campos adicionais no formulário de atributos, optar entre três interfaces de edição e aprender sobre o funcionamento das ferramentas através de um tutorial oferecido na primeira edição.

O Wikimapia trabalha com dois fluxos de ações separados: no primeiro, em poucos passos, o usuário pode inserir livremente as feições e os seus atributos, sem a necessidade de autenticação. No segundo fluxo, o usuário precisa estar necessariamente autenticado ao sistema para que possa editar uma feição pré-existente e seus atributos. A partir deste ponto tem acesso a todas as funcionalidades de desenho e edição. O tipo de feição que pode ser inserida depende do tempo de cadastro do usuário, bem como da sua experiência, associada a sua identificação no sistema (por exemplo, um usuário anônimo ou cadastrado recentemente tem a permissão para apenas inserir feições de área, seus atributos e novas localidades).

O Sistema Wikiloc tem um fluxo de ações encadeadas e orienta o usuário a escolher a forma de inserção da feição (através do carregamento de um arquivo GPX ou pelo desenho direto sobre o mapa), exige a autenticação do usuário e pergunta ao usuário por meio de um diálogo, qual a localização aproximada da feição (no caso trilha) a ser inserida. Após a inserção da feição, o usuário é direcionado à tela de atributos do conjunto de feições inseridas.

Um aspecto comum aos três sistemas é a obrigatoriedade do usuário de executar uma função de salvamento do desenho ou edição. Nenhum dos sistemas possui uma função de salvamento temporário ou avisa ao usuário para que este salve a feição. 


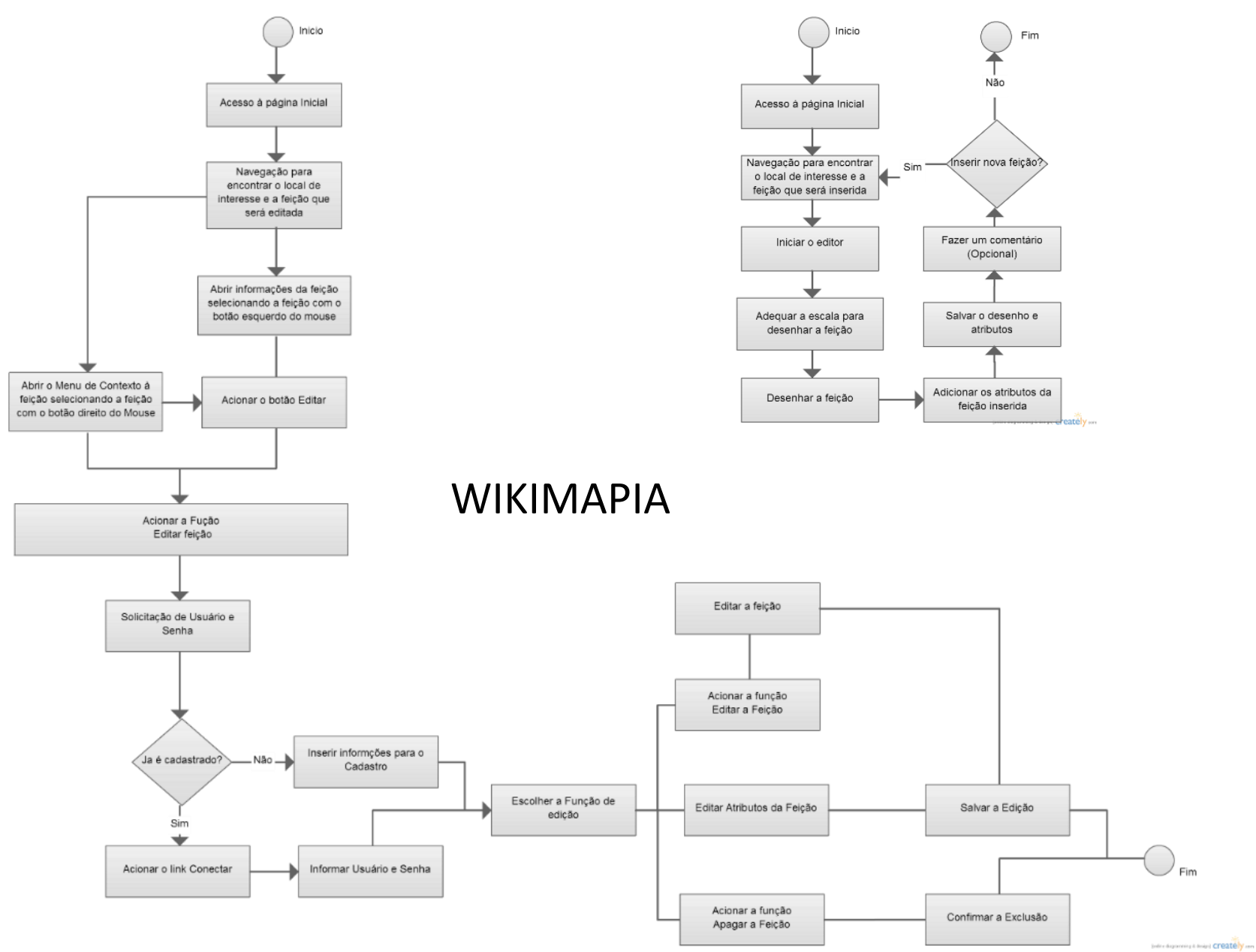

Figura 5 : Fluxograma de desenho e edição de feições no Wikimapia.

\subsection{Perfil dos Usuários Testados}

Quanto ao perfil dos usuários, 90\% deles têm idades entre 17 e 34 anos, todos possuem a escolaridade mínima de nível superior. Quanto ao perfil de uso da Internet, 97\% dos usuários usam frequentemente a Internet, sendo que 37\% estão sempre conectados, $56 \%$ acessam a Internet via um computador pessoal ou um smartphone, mas $43 \%$ acessam via ambos os dispositivos.

Todos os usuários entrevistados relataram que são usuários de mapas na Internet e metade deles usa com muita frequência esses mapas. Quando perguntados se utilizam alguma aplicação ou aplicativo móvel para acesso aos mapas na Internet, 70\% dos usuários disseram que sim. Desses usuários, $69 \%$ responderam utilizar o GoogleMaps.

\subsection{Primeiro Contato com o Sistema}

O objetivo de uma aplicação deve ser comunicado rapidamente ao usuário durante a sua primeira visita, de modo a manter seu interesse e evitar que ele migre a um outro site. Por isso, na tarefa 1 , os usuários foram testados se seriam capazes de entender os objetivos do site nos 5 minutos 
dados para a tarefa. Foi pedido que os indivíduos testados selecionassem entre mapeamento, navegação, rede social e turismo, como o objetivo principal do site testado. As categorias "rede social" e "turismo" não foram escolhidas aleatoriamente, mas devido a características dos sistemas que permitem a interação entre usuários do sistema, a associação das feições com atividades ao ar livre ou uso turístico, e até premiam os feitos de seus usuários.

O resultado foi que 8 usuários, correspondente a $27 \%$ do total testado, entenderam que os objetivos dos sites estão relacionados a turismo e/ou redes sociais, o que reflete uma dissonância cognitiva derivada dos elementos de comunicação das interfaces, o que pode refletir na permanência dos usuários na ferramenta e/ou na qualidade e pertinência das feições inseridas.

Quando perguntados sobre informações adicionais do sistema, como "Ajuda e Documentação", que auxiliam os usuários no seu uso, 11 dos os indivíduos testados disseram não encontrar uma área especifica de Ajuda, apesar de terem respondido anteriormente que os sites possuíam documentação ao usuário. Isso evidencia uma possível falta de entendimento das informações dispostas ou até o desuso dessas ferramentas de auxílio, o que pontua uma maior importância nas soluções de interface e usabilidade.

É necessário testar como os usuários percebem, leem e interpretam os elementos gráficos e textuais das interfaces dos sites, pois, no caso dos sites testados, a associação dos objetivos dos sites ao turismo e as redes sociais, bem como aqueles que não encontraram as áreas de ajuda aos usuários, contradizem os $97 \%$ que consideraram as informações nos sites como claras e inteligíveis.

Relacionado ao público alvo dos sistemas web, a maioria dos usuários (56\%) consideraram que as aplicações testadas se destinavam a qualquer pessoa, contra $44 \%$ que entenderam os sites como voltados somente à usuários com mais conhecimento em mapas na Internet. Isso evidencia também um possível erro de comunicação sobre os objetivos e público dos sites, assim como pode ter relação ao grau de dificuldade que esses usuários vivenciaram durante a execução dos testes. Não foi possível a identificação exata dessa confusão de público devido ao número pequeno de usuários testados.

\subsection{Debriefing}

No questionário $\mathrm{C}$, aplicado imediatamente após os usuários executarem as tarefas solicitadas nos testes, foi solicitado que os indivíduos testados indicassem um grau de dificuldade geral na execução das tarefas solicitadas. A escala de dificuldade ia de 0 (zero) à 10 (dez), em que zero correspondia a uma experiência extremamente fácil e dez correspondia a uma experiência extremamente difícil.

Quando perguntados sobre a dificuldade geral percebida na execução das tarefas, o sistema OSM foi considerado moderado a fácil, assim como o Wikimapia e moderada a difícil para os usuários que testaram o site Wikiloc. A percepção do usuário é importante para evidenciar como os elementos de interação e usabilidade podem estar influenciando a experiência do uso.

\subsubsection{Localização da área de estudo}

A localização da área de estudo onde seriam desenhadas as feições era essencial para a execução da tarefa, bem como idêntica a situação real de colaboração. Para tanto, os usuários tinham a possibilidade de usar um método de pesquisa textual ou de localizá-la interativamente navegando 
sobre o mapa. Quando perguntados sobre a dificuldade de localizar a região do teste, informada pelo enunciado da tarefa, a maioria dos usuários que testaram o Wikimapia entenderam como extremamente fácil a localização da área de estudo, situação não compartilhada pelos usuários que testaram o Wikiloc e principalmente pelos usuários que testaram o OSM que acharam difícil a sua localização.

Para a busca de evidencias que justificassem a diferença supracitada, foram utilizadas as gravações feitas durante os testes. Foi constatado que todos os usuários localizaram a área de estudo, mas metade dos usuários que testaram o OSM utilizaram a função de pesquisa, enquanto 90\% dos usuários que testaram o Wikimapia localizaram a área de estudo interativamente através do uso do mouse, isto sugere que a interface do site Wikimapia estimula mais a interação dos usuários na tarefa de localizar uma posição geográfica específica que o site OSM. No caso do Wikiloc, o site permite localizar a área apenas por meio de pesquisa textual através do nome.

A grande diferença entre os sistemas é que o Wikimapia possui a funcionalidade de dicas sobre as feições, em outras palavras permite que o usuário, ao passar o cursor do mouse sobre uma feição existente, obtenha informações instantâneas.

\subsubsection{Função de inserção (desenho) de feições}

A função de edição, objetivo principal desta pesquisa, foi encontrada facilmente pela maioria dos usuários, mas alguns dos usuários que testaram o site Wikimapia consideraram difícil a sua localização. A interface do OSM oferece um botão/menu para iniciar a interface de edição. Nesse botão/menu o usuário pode iniciar diretamente a função de edição principal ou abrindo o menu, escolher entre outras duas alternativas de interface. Todos os usuários escolheram a primeira opção de interface de edição, mas $70 \%$ deles não pressionaram diretamente a função, optaram por abrir o diálogo do menu para escolher a função de edição.

No caso do Wikiloc $60 \%$ dos usuários acharam fácil encontrar a função de edição, mas percebeu-se através das gravações que $40 \%$ dos usuários iniciaram a edição acidentalmente, pois não perceberam que ela já estava ativa ao migrarem à interface de edição.

\subsubsection{Localização da feição geográfica}

A identificação da feição que seria desenhada foi considerada pela maioria dos usuários do OSM como moderadamente difícil à difícil, enquanto o mesmo número de usuários do Wikimapia considerou fácil à extremamente fácil encontrar a feição. Essa diferença de opinião, tão expressiva, entre esses dois grupos de usuários pôde ser explicada também pelas gravações efetuadas; nelas os usuários do OSM perdiam a referência espacial quando a interface de edição era carregada, pois havia a mudança de escala e visualização de um mapa vetorial para uma imagem de satélite. Os usuários precisavam se localizar novamente, enquanto no Wikimapia isso não acontecia.

\subsubsection{Processo de Inserção}

A Tabela 3 traz algumas impressões dos usuários quanto ao processo de inserção, bem como alguns aspectos observados através dos vídeos dos testes. 
Tabela 3: Observações sobre os testes

\begin{tabular}{|l|}
\hline OPENSTREETMAP: DIFICULDADE MODERADA À FÁCIL \\
\hline A existência de Tool tips e atalhos ajudou os usuários \\
\hline A posição dos botões é de fácil localização \\
\hline Os símbolos desenhados nos botões comunicam claramente o que a função faz \\
\hline Interface com layout limpo e preciso. \\
\hline A autenticação é requerida em todos os processos (desenho e edição) \\
\hline WIKIMAPIA: DIFICULDADE MODERADA À DIFÍCIL \\
\hline A autenticação não é obrigatória para a inserção de feições \\
\hline $\begin{array}{l}\text { A autenticação do usuário durante o processo de desenho ou edição confunde o usuário, pois inicia } \\
\text { com o formulário de cadastro. }\end{array}$ \\
\hline Nenhum usuário usou os botões de contexto de edição no canto superior direito da interface. \\
\hline A distância entre o botão Salvar e o local de edição causou confusão aos usuários. \\
\hline $60 \%$ dos usuários não entenderam em um primeiro momento como finalizar o desenho de uma feição. \\
\hline $100 \%$ dos usuários posicionaram a região de estudo na porção central inferior da interface de mapa. \\
\hline Nenhum usuário acionou o menu que permite alternar entre mapas base. \\
\hline Possui tooltips. \\
\hline WIKILOC: DIFICULDADE MODERADA \\
\hline 40\% dos usuários iniciaram acidentalmente o desenho da feição. \\
\hline Não é claro aos usuários quando o desenho da feição foi finalizado. \\
\hline Não existem tooltips \\
\hline Nenhum usuário usou a função tela cheia disponível na interface. \\
\hline
\end{tabular}

\subsubsection{Edição e exclusão da feição desenhada}

$\mathrm{Na}$ atividade de edição e exclusão das feições inseridas, $80 \%$ dos usuários do OSM declararam como sendo moderado à fácil o nível de dificuldade ao editar a feição e em seguida apagá-la, apesar disso, 30\% dos usuários testados não conseguiram concluir a tarefa. Esta diferença pode evidenciar uma dissonância entre o que é comunicado ao usuário do sistema e o que é efetivamente entendido e executado por ele.

No Wikimapia, 60\% dos usuários acharam as atividades de editar uma feição existente e de apagar uma feição de moderado à difícil. Metade dos usuários utilizaram o menu de contexto para iniciar a edição e apagar a feição, enquanto a outra metade acessou a funcionalidade via outros menus da interface. Isso pode ser a evidencia que um dos métodos de edição e exclusão não são bem recebidos pelos usuários.

A diferença entre o resultado do OSM e do Wikimapia, nos testes com as tarefas de inserção, edição e exclusão de feições, pode ter relação principalmente com as heurísticas de usabilidade de (i) Consistência e Padrões, (ii) Reconhecer em vez de relembrar, (iii) Flexibilidade e eficiência de uso e (iv) Design estético e minimalista. Isto porque o OSM se mostrou mais aderente a esses padrões, mantendo as funções em destaque em relação ao mapa, mas agradáveis aos olhos; sempre nas mesmas posições, facilitando a sua localização; processualmente simples de usar e mantendo a consistência de padrões em todo o site. 


\subsection{Avaliação através das Heurísticas de Usabilidade}

O resultado da análise quanto ao atendimento das heurísticas de usabilidade está disposto no Tabela 4, relacionando as heurísticas a cada um dos sistemas VGI testados.

Tabela 4 : Análise do atendimento as Heurísticas de usabilidade

\begin{tabular}{|l|c|c|c|}
\hline \multicolumn{1}{|c|}{ Heurísticas de Usabilidade } & OSM & Wikimapia & Wikiloc \\
\hline Visibilidade do estado do sistema & ++ & ++ & -- \\
\hline Mapeamento entre o sistema e o mundo real & ++ & +- & ++ \\
\hline Liberdade e controle do usuário & ++ & +- & +- \\
\hline Consistência e Padrões & +- & -- & ++ \\
\hline Prevenção de erros & ++ & +- & ++ \\
\hline Reconhecer em vez de relembrar & ++ & +- & ++ \\
\hline Flexibilidade e eficiência de uso & ++ & +- & ++ \\
\hline Design estético e minimalista & ++ & +- & ++ \\
\hline $\begin{array}{l}\text { Suporte para o usuário reconhecer, diagnosticar } \\
\text { e recuperar erros }\end{array}$ & ++ & ++ & +- \\
\hline Ajuda e documentação & ++ & ++ & +- \\
\hline
\end{tabular}

\section{Conclusões}

Através da análise dos questionários e gravações dos testes foi constatado que no primeiro contato com as interfaces dos sistemas VGI, antes de explorar os elementos da interface, os usuários tentam encontrar no mapa um local conhecido, como a sua residência, um local que costuma frequentar ou pretende visitar. Da mesma maneira, a interação via o mouse pareceu natural para todos os usuários, intuitiva e decorrente de um estímulo automático.

As interfaces minimalistas e de cores claras, como do OSM, mantém os usuários mais confortáveis, os estimulam a explorar os menus e elementos da interface, e os mantém focados durante a execução de uma atividade, ao contrário da interface do Wikimapia, escuras e densas de informação. Além disso, a variação no tamanho da área do mapa, durante a execução das feições, confunde o usuário e muitas vezes é subutilizada.

Elementos de interface, como botões e menus, referentes a mesma funcionalidade do sistema, dispostos simultaneamente na interface, confundem um usuário novo ou iniciante. As funções que não estão disponíveis para a execução dos usuários, não devem ter seus links na interface. Os sites que utilizam linguagens mais próximas a realidade dos usuários, como "Editar" em oposição a "Adicionar local", tem as funções encontradas mais facilmente e as tarefas executadas mais rapidamente. Isso foi verificado em todos os sistemas testados.

Devem-se obter novas formas de orientar o usuário quanto a utilização dos sistemas, já que a maioria dos usuários testados não utilizaram a documentação existente. No caso do site OSM, o 
tutorial interativo disponível no primeiro momento de acesso à interface de edição, também não se mostrou atrativo aos usuários.

No site Wikimpaia, as dicas sobre as feições e localidades, que aparecem no mapa quando o usuário passa o cursor do mouse sobre a feição, e as toponímias parecem auxiliar o usuário a estabelecer uma referência espacial e a localizar mais facilmente as feições sobre o mapa. Essa funcionalidade estabelece um estímulo espacial mais rápido e direto com o usuário e podem ser estudadas novas possibilidades de seu uso.

Foi constatado que os usuários posicionam o local de inserção ou edição de uma feição em uma posição confortável aos olhos na tela, em geral a porção inferior central do frame do mapa. A posição e visibilidade dos botões e menus em relação ao local onde as feições são desenhadas ou editadas tem impacto no seu uso, assim como na execução e conclusão da tarefa (ex.: a função salvar distante do desenho).

No contexto desta pesquisa, sugere-se como continuação e futuros trabalhos, a expansão do número de usuários testados para a confirmação das evidencias listadas acima, o aperfeiçoamento da metodologia de teste empregada, a ampliação das funcionalidades de interfaces VGI testadas e o teste de interfaces de sistemas VGI para dispositivos móveis.

\section{AGRADECIMENTOS}

Os autores agradecem ao CNPq pelo auxílio financeiro. - Processos 459300/2014-8 e 301980/2014-4 e pela bolsa de doutorado concedida ao primeiro autor.

\section{REFERENCIA BIBLIOGRÁFICA}

Brown, M. Sharples, S. Harding, J. Parker, C.J. Bearman, N. Maguire, M. Forrest, D. Haklay, M. Jackson, M. Usability of Geographic Information: Current challenges and future directions, Applied Ergonomics, Volume 44, Issue 6, November 2013, Pages 855-865, ISSN 0003-6870.

Cybis, Walter de Abreu. Ergonomia e usabilidade: conhecimentos, métodos e aplicações. 2. ed. rev. e ampl. São Paulo: Novatec, 2010. 422 p.

Goodchild, M.F. Citizens as sensors: the world of volunteered geography. GeoJournal n 69 (4): p. 211-221. 2007.

ISO 9241-11, 1998. Ergonomic requirements for office work with visual display terminals (VDT)s - Part 11 Guidance on usability.

Maziero, L.T.P. Influência dos aspectos das interfaces na comunicação dos mapas interativos e a proposição de diretrizes para o design dessas interfaces. Tese de Doutorado - Curso de Pósgraduação em Ciências Geodésicas. Universidade Federal do Paraná, 2007.

Mendonça, A. Avaliação de interfaces para mapas funcionais na web. 2009. 185f. Dissertação (mestrado) - Universidade Federal do Paraná, Setor de Ciências da Terra, Programa de PósGraduação em Ciências Geodésicas. Defesa: Curitiba, 27/07/2009. 
Nielsen, J. \& Loranger, H. Usabilidade na Web: projetando websites com qualidade. Rio de Janeiro: Elsevier, 2007. xxiv, 406 p., il. Inclui índice. ISBN 9788535221909 (broch.).

Nielsen, J. 10 Usability Heuristics for User Interface Design. Nielsen Norman Group Website Articles, 1995. Disponível em <http://www.nngroup.com/articles/ten-usability-heuristics/> Acesso em 10/08/2014

Nielsen, J. Usability Engineering. Oxford: Academic Press, 1993

Openstreetmap. Openstreetmap. Disponível em: http://www.openstreetmap.org/ . Acesso em: $12 / 10 / 2014$

O'reilly, T. What is Web 2.0: design patterns and business models for the next generation of software. Disponível em: <http://www.oreillynet.com/pub/a/oreilly/tim/news/2005/09/30/whatis-web-20.html>. . Acesso em: 03/01/2016

Parker, C. The Fundamentals of Human Factors Design for Volunteered Geographic Information. London, UK: Springer, 2014. 137 p., ISBN 978-3-319-03503-1 (eBook).

Tulloch, D.L. Is VGI participation? From vernal pools to video games". GeoJournal, Vol. 72, pp. 161-171. 2008.

Wikiloc. Wikiloc. Disponível em: http://www.wikiloc.com/ . Acesso em:12/10/2014

Wikimapia. Wikimapia. Disponível em: http://wikimapia.org/about/. Acesso em: 13/10/2014.

Recebido em Setembro de 2015.

Aceito em Fevereiro de 2016. 